\title{
Stents Liberadores de Sirolimus com e sem Cobertura Polimérica: Análise Seriada com Angiografia e Ultrassom Intracoronariano Tridimensional
}

\author{
Daniel Chamié ${ }^{1}$, José Ribamar Costa Jr. ${ }^{1}$, Alexandre Abizaid ${ }^{1}$, José Fábio Almiro da Silva ${ }^{1}$, Fausto Feres ${ }^{1}$, \\ Luiz Alberto Mattos', Rodolfo Staico', Ricardo A. Costa', Dimytri Siqueira', Luiz Fernando Tanajura', \\ Amanda G. M. R. Sousa', J. Eduardo Sousa ${ }^{1}$
}

\section{RESUMO}

Introdução: A presença de polímeros sintéticos em contato com a parede vascular tem sido associada a intensa resposta inflamatória local e a eventos adversos tardios. Entretanto, sua ausência pode comprometer a eficácia dos stents farmacológicos em suprimir a hiperplasia intimal. Avaliamos, com angiografia coronariana quantitativa (ACQ) e ultrassom intracoronariano (USIC), a eficácia do stent Vestasync $^{T M}$ (VES), liberador não-polimérico de sirolimus em baixa dose $(55 \mu \mathrm{g})$, e do stent Cypher ${ }^{\mathrm{TM}}(\mathrm{CYP})$, liberador de sirolimus polimérico em alta dose $(140 \mu \mathrm{g})$. Método: Quinze pacientes com lesões primárias < $14 \mathrm{~mm}$, em artérias coronárias nativas de $2,5 \mathrm{~mm}$ a $3,5 \mathrm{~mm}$, foram tratados com VES e comparados a uma coorte histórica de 15 pacientes tratados com CYP. ACQ e USIC foram realizados pós-procedimento, aos 4 e 12 meses. Desfechos primários foram: perda tardia intrastent e porcentual de obstrução volumétrica intrastent. Resultados: As características clínicas eram semelhantes. O grupo VES tinha vasos menores $(2,67 \pm 0,32 \mathrm{~mm}$ vs. $2,98 \pm 0,4 \mathrm{~mm} ; \mathrm{P}=0,02)$. Aos 4 meses, o grupo VES mostrou tendência a maior perda tardia intrastent $(0,30 \pm 0,25 \mathrm{~mm}$ vs. $0,10 \pm 0,3 \mathrm{~mm}$; $P=0,06)$, mas porcentual de obstrução volumétrica intrastent similar ao do grupo CYP $(2,8 \pm 2,2 \%$ vs. $2,2 \pm$ $3,8 \% ; \mathrm{P}=0,60)$. Aos 12 meses, a perda tardia intrastent foi de $0,37 \pm 0,24 \mathrm{~mm}$ no grupo submetido a implante de stent VES vs. $0,16 \pm 0,36 \mathrm{~mm}$ no grupo submetido a implante de stent CYP $(P=0,074)$, e o porcentual de obstrução volumétrica intrastent foi de $4,0 \pm 2,2 \%$ no grupo que recebeu stent VES vs. $2,4 \pm 3,9 \%$ no grupo que recebeu stent CYP $(P=0,17)$. Não houve casos de reestenose binária. Conclusões: $O$ stent VES mostrou-se seguro e eficaz em suprimir a hiperplasia intimal até 12 meses. Ausência do polímero e dosagem reduzida da droga não comprometeram sua eficácia. Estudos maiores e com

\author{
ABSTRACT \\ Sirolimus-Eluting Stents with and without Polymeric \\ Coating: Serial Angiography and Three-dimensional \\ Intravascular Ultrasound Analysis
}

Background: The long-term presence of the stent polymer in contact with the vessel wall has been associated to intense inflammatory response and late adverse events. However, absence of the stent polymer may compromise drug dose and drug delivery kinetics, leading to some efficacy issues. We aimed to compare, by means of serial quantitative coronary angiography (QCA) and intravascular ultrasound (IVUS), the efficacy of the novel nonpolymeric low-dose $(55 \mu \mathrm{g}) \mathrm{VESTAsync}^{\mathrm{TM}}$ (VES) sirolimus-eluting stent, with the permanent polymer, high-dose $(140 \mu \mathrm{g})$ Cypher $^{\mathrm{TM}}$ sirolimus-eluting stent (CYP). Methods: Fifteen patients with single, de novo lesions $<14 \mathrm{~mm}$, in native vessels of $2.5 \mathrm{~mm}$ to $3.5 \mathrm{~mm}$ diameter, were treated with VES and compared to a paired historical cohort of 15 patients treated with CYP. QCA and IVUS data were obtained post-procedure and at 4 and 12-month follow-up. Primary endpoints were the comparison of in-stent late luminal loss and percentage of intimal hyperplasia obstruction. Results: Clinical characteristics were similar between groups. The VES group had smaller vessels $(2.67 \pm 0.32 \mathrm{~mm}$ vs. $2.98 \pm 0.4 \mathrm{~mm}$; $\mathrm{P}=0.02)$. At 4 months, the $\mathrm{VES}$ group had a tendency towards a higher in-stent late luminal loss $(0.30 \pm 0.25 \mathrm{~mm}$ vs. $0.10 \pm 0.3 \mathrm{~mm} ; \mathrm{P}=0.06)$ but similar IVUS $\%$ of intimal hyperplasia obstruction $(2.8 \pm 2.2 \%$ vs. $2.2 \pm 3.8 \% ; \mathrm{P}=0.60)$. At 12 -months, in-stent late luminal loss was $0.37 \pm 0.24 \mathrm{~mm}$ (VES) vs. $0.16 \pm 0.36 \mathrm{~mm}(\mathrm{CYP}), \mathrm{P}=0.074$, and the IVUS \% of intimal hyperplasia obstruction was $4.0 \pm 2.2 \%$ (VES) vs. $2.4 \pm 3.9 \%(C Y P), P=0.17$. There were no cases of binary restenosis. Conclusion: The VES proved safe and effective in reducing intimal hyperplasia formation up to 12

\footnotetext{
1 Instituto Dante Pazzanese de Cardiologia - São Paulo, SP, Brasil. Correspondência: Alexandre Abizaid. Instituto Dante Pazzanese de Cardiologia. Av. Dr. Dante Pazzanese, 500 - 14o andar - São Paulo, SP, Brasil - CEP 04012-180 E-mail: aabizaid@uol.com.br

Recebido em: 9/12/2008 • Aceito em: 23/2/2009
} 
seguimento mais longo são necessários para avaliar se esses achados melhorarão o perfil de segurança dos stents farmacológicos.

DESCRITORES: Contenedores. Stents farmacológicos. Sirolimo. Angiografia. Ultrassom. months. The absence of the polymer, as well as the lower drug dose, did not impact the efficacy of this device. Whether these changes will affect the safety profile of this novel DES has to be confirmed in a larger cohort of patients and longer follow up.

DESCRIPTORS: Stents. Drug-eluting stents. Sirolimus. Angiography. Ultrasonics.
0 advento dos stents farmacológicos promoveu efetiva inibição do processo de proliferação neointimal, resultando em expressiva redução das taxas de reestenose intrastent e da necessidade de nova revascularização da lesão-alvo $0^{1-4}$. Os excelentes resultados obtidos nos diferentes subgrupos de pacientes tornaram esses dispositivos os mais utilizados para procedimentos de intervenção coronária percutânea em muitos centros 5 .

Contudo, seguimento clínico mais longo desses pacientes tem revelado preocupações com relação à segurança tardia desses dispositivos, a partir de relatos de trombose $\mathrm{e}^{6-8}$ e reestenose $\mathrm{e}^{9,10}$ tardias. Estudos anatomopatológicos têm demonstrado que esses fenômenos estão associados a reações inflamatórias prolongadas ${ }^{10-12}$ e que a presença de um polímero sintético em contato com a parede vascular é o principal mecanismo responsável ${ }^{10,11}$.

Nesse sentido, tem havido crescente interesse no desenvolvimento de novos stents farmacológicos, com diferentes tecnologias para liberação do fármaco sem a presença do polímero. Entretanto, a ausência do polímero na composição dos stents farmacológicos, responsável pelo carreamento e pela liberação programada dos agentes antiproliferativos, poderia alterar a cinética de liberação dos medicamentos e afetar a eficácia desses dispositivos. De fato, estudos que avaliaram stents farmacológicos sem cobertura polimérica têm apresentado resultados de efetividade conflitantes ${ }^{13-17}$.

O stent VESTAsync ${ }^{\text {TM }}$ (MIV Therapeutics Inc., Atlanta, Estados Unidos) é um novo stent farmacológico que promove a liberação do fármaco sirolimus a partir da modificação da superfície de suas hastes metálicas, com uma cobertura nanoporosa de hidroxiapatita, sem a presença de polímero ${ }^{18}$. Este estudo teve como objetivo apresentar os resultados de efetividade desse stent farmacológico de nova geração, comparativamente aos resultados de uma série histórica de pacientes submetidos a implante do stent Cypher ${ }^{\mathrm{TM}}$ (Cordis Corp., Johnson \& Johnson, Miami Lakes, Estados Unidos), também liberador de sirolimus, por meio de cobertura polimérica durável.

\section{MÉTODO}

\section{Desenho do estudo}

Este é um estudo prospectivo, não-randomizado e unicêntrico, que visa à comparação, por meio de análise angiográfica e ultrassom intracoronariano seriados, da efetividade do stent VESTAsync ${ }^{\mathrm{TM}}$ com a do stent Cypher $^{\mathrm{TM}}$, utilizado em uma coorte histórica de pacientes portadores de doença arterial coronariana.

\section{Seleção de pacientes}

Durante o mês de maio de 2007, 15 pacientes portadores de doença arterial coronariana, com lesões únicas, de novo, de extensão menor que $14 \mathrm{~mm}$, em artérias coronárias nativas, de diâmetro entre $2,5 \mathrm{~mm}$ e $3,5 \mathrm{~mm}$, foram tratados com o implante do stent VESTAsync ${ }^{\mathrm{TM}}$. Foram excluídos os pacientes portadores de infarto do miocárdio nos últimos 30 dias, lesões no tronco da artéria coronária esquerda e/ou lesões ostiais ou em bifurcações, bem como oclusões crônicas. Pacientes portadores de disfunção ventricular esquerda (fração de ejeção ventricular esquerda $<30 \%$ ) e disfunção renal (creatinina sérica $>2,0 \mathrm{mg} / \mathrm{dl}$ ) significativas também foram excluídos.

O grupo controle foi constituído de 15 pacientes, com os mesmos critérios de inclusão e exclusão, pareados com relação às características clínicas e angiográficas de base, tratados com o stent Cypher ${ }^{\mathrm{TM}}$ em 2000 .

O protocolo do estudo foi aprovado pelo Comitê de Ética em Pesquisa de nossa instituição, e termo de consentimento informado foi assinado e fornecido por todos os pacientes incluídos na análise.

\section{Características dos dispositivos estudados}

\section{Stent VESTAsync ${ }^{T M}$}

A plataforma metálica é o stent GenX, de aço inoxidável (316 L) e geometria variável, com hastes de fina espessura $(105 \mu \mathrm{m})$, pré-montado em um cateterbalão de poliamida. Suas hastes são modificadas e recobertas por uma fina camada nanoporosa $(500 \mathrm{~nm}$ a $700 \mathrm{~nm}$ ) de hidroxiapatita, responsável pelo carreamento e pela liberação do fármaco, sem necessidade da aplicação de polímeros sintéticos.

A hidroxiapatita é uma substância naturalmente encontrada no corpo humano, principalmente na constituição dos ossos e do esmalte dos dentes, apresentando excelente biocompatibilidade e ausência de toxicidade, com o potencial de não deflagrar reações adversas produzidas pelo sistema imune ${ }^{19-21}$. Estudos 
Chamié D, et al. Stents Liberadores de Sirolimus com e sem Cobertura Polimérica: Análise Seriada com Angiografia e Ultrassom Intracoronariano Tridimensional. Rev Bras Cardiol Invas. 2009;17(1):20-30.

clínicos demonstraram que nanocristais de hidroxiapatita, quando aplicados ao território vascular, mantêm marcadores morfológicos e bioquímicos de um funcionamento saudável das células endoteliais, sem a aquisição de fenótipos pró-inflamatórios ${ }^{21}$, comumente $^{2}$ associados com as coberturas poliméricas utilizadas em stents farmacológicos. A Figura 1 apresenta imagens de fotomicrografia eletrônica com aspectos da cobertura de hidroxiapatita, bem como a mesma carregada com a formulação do fármaco sirolimus.

O fármaco liberado é o sirolimus, um antibiótico macrolídeo com propriedades imunossupressoras, aprovado pelo Food and Drug Administration (FDA), em 1999, para profilaxia de rejeição ao transplante renal $^{22}$. O sirolimus liga-se à proteína citosólica FKbinding protein 12 (FKBP12), e esse complexo inibe a mammalian target of rapamycin (mTOR), bloqueando a progressão do ciclo celular da fase G1 para S. A dose do sirolimus aplicada é de $55 \mu \mathrm{g}$ por $19 \mathrm{~mm}$ de stent $(2,9 \mu \mathrm{g} / \mathrm{mm})$, com cerca de $80 \%$ dela liberada em 60 dias.

No stent VESTAsync ${ }^{\mathrm{TM}}$, o sirolimus é carreado em uma formulação lipofílica, que preenche as porosidades da cobertura de hidroxiapatita, sem acrescentar à espessura das hastes. Após aplicação da cobertura de hidroxiapatita com sirolimus, a espessura total das hastes do stent VESTAsync ${ }^{\mathrm{TM}}$ é de $106 \mu \mathrm{m}$.

\section{Stent Cypher ${ }^{T M}$}

O stent Cypher ${ }^{\mathrm{TM}}$ utilizado nesta análise era constituído por uma plataforma metálica de aço inoxidável (Bx Velocity ${ }^{\mathrm{TM}}$ ), recoberto por uma combinação de dois polímeros, o polyethylene-co-vinyl acetate (PEVA) e o poly n-butyl methacrylate (PBMA), carregados com o

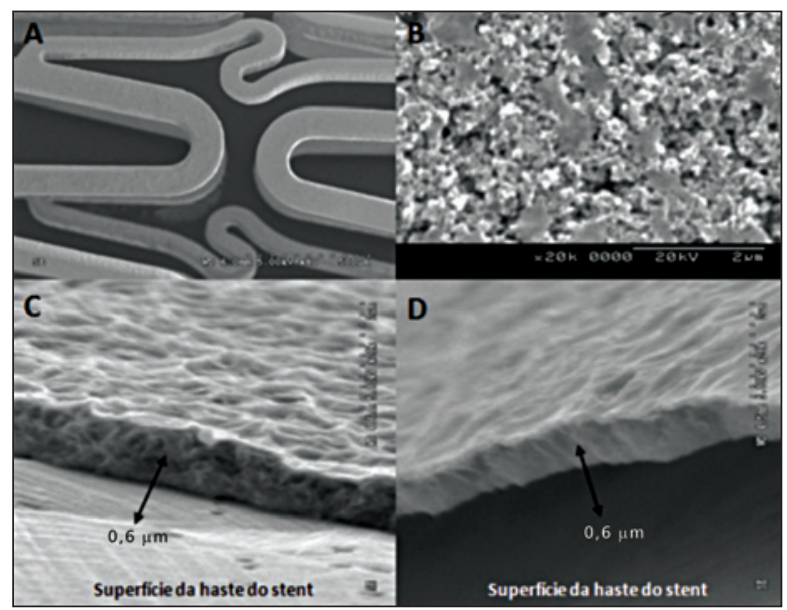

Figura 1 - Imagens de microscopia eletrônica dos componentes do stent VESTAsync ${ }^{T M}$. Em A, detalhe do desenho das hastes do stent Gen $X^{T M}$. Em B, a cobertura nanoporosa de hidroxiapatita. Em C, detalhe da cobertura de hidroxiapatita sobre as hastes do stent. Em D, imagem da cobertura final da superfície nanoporosa de hidroxiapatita carregada com formulação lipofílica de sirolimus. fármaco sirolimus, na dose de $140 \mu \mathrm{g}$ por $18 \mathrm{~mm}$ de stent $(7,7 \mu \mathrm{g} / \mathrm{mm})$. Em cerca de 30 dias, 80\% da dose total do sirolimus é liberada.

Depois de aplicadas as coberturas dos polímeros e do fármaco, a espessura total das hastes do stent Cypher $^{\mathrm{TM}}$ é de $154 \mu \mathrm{m}$.

Para fins de comparação, apresentamos, na Figura 2, as curvas de eluição do sirolimus nos stents VESTAsync $^{T M}$ e Cypher ${ }^{T M}$.

A Tabela 1 resume as principais características dos dois stents.

\section{Procedimento de intervenção coronária}

Todos os pacientes foram pré-tratados com aspirina $300 \mathrm{mg} 24$ horas antes do procedimento (caso não estivessem usando previamente) e um tienopiridínico em dose de ataque. A ticlopidina era administrada na dose de $500 \mathrm{mg}$ por dia pelo menos 48 horas antes do procedimento e o clopidogrel, na dose de 300 mg, 24 horas antes. Após o procedimento, a terapia de antiagregação plaquetária dupla foi mantida pelo período mínimo de 6 meses para todos os pacientes, com o clopidogrel ou a ticlopidina suspensos a partir de então, e a aspirina mantida indefinidamente.

Durante o procedimento, todos os pacientes receberam heparina não-fracionada na dose de $100 \mathrm{UI} / \mathrm{kg}$, visando à manutenção do tempo de coagulação ativada entre 250 e 350 segundos. O uso de inibidores da glicoproteína IIb/IIla não era obrigatório e ficou a critério do operador.

A angiografia inicial era realizada em pelo menos duas projeções ortogonais, após administração de $100 \mu \mathrm{g}$ a $200 \mu \mathrm{g}$ de nitroglicerina intracoronariana.

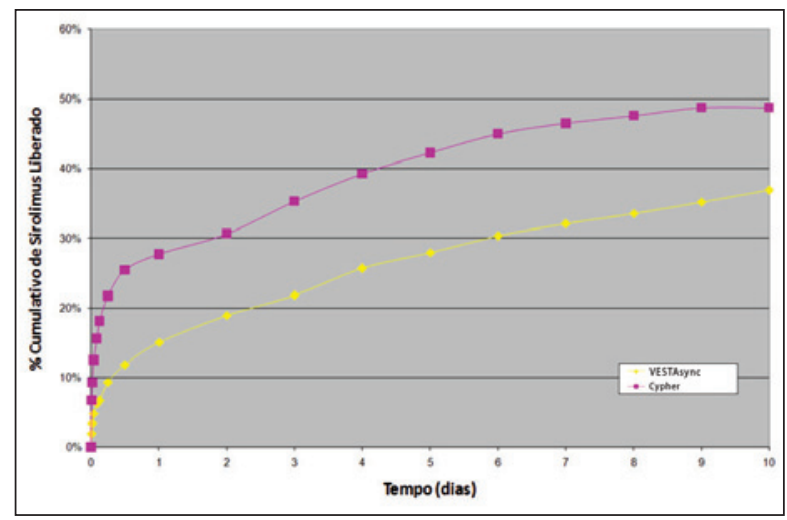

Figura 2 - Gráfico ilustrativo das curvas de eluição cumulativa, in vitro, do sirolimus, até o tempo de 10 dias. Na primeira hora, a liberação do sirolimus pelo stent VESTAsync ${ }^{T M}$ é semelhante à do stent Cypher $^{\mathrm{TM}}$. A partir de então, a taxa de liberação da droga pelo VESTAsync $^{\text {TM }}$ cai à metade, com sua curva de eluição correndo em paralelo à do Cypher $^{\mathrm{TM}}$. Em 30 dias, 80\% do fármaco já foi liberado pelo stent Cypher $^{\mathrm{TM}}$, enquanto o VESTAsync ${ }^{\mathrm{TM}}$ necessita 60 dias para liberação do mesmo porcentual do fármaco. 
TABELA 1

Principais características dos stents VESTAsync ${ }^{\mathrm{TM}}$ e Cypher $^{\mathrm{TM}}$

\begin{tabular}{|c|c|c|}
\hline & VESTAsync $^{\mathrm{TM}}$ & Cypher ${ }^{\mathrm{TM}}$ \\
\hline \multicolumn{3}{|l|}{ PLATAFORMA } \\
\hline Nome & GenXTM & Bx Velocity ${ }^{\mathrm{TM}}$ \\
\hline Material & Aço inoxidável & Aço inoxidável \\
\hline Desenho & 10 células/geometria variável & Células fechadas \\
\hline \multicolumn{3}{|l|}{ FÁRMACO } \\
\hline Fármaco antiproliferativo & Sirolimus & Sirolimus \\
\hline Dose & $55 \mu \mathrm{g} / 19 \mathrm{~mm}$ de stent $(2,9 \mu \mathrm{g} / \mathrm{mm})$ & $140 \mu \mathrm{g} / 18 \mathrm{~mm}$ de stent $(7,7 \mu \mathrm{g} / \mathrm{mm})$ \\
\hline Tempo de eluição & $80 \%$ em 60 dias & $80 \%$ em 30 dias \\
\hline \multicolumn{3}{|l|}{ POLÍMERO } \\
\hline Nome & $\begin{array}{l}\text { Sem polímero } \\
\text { Modificação da superfície das hastes } \\
\text { com cobertura nanoporosa de HAp }\end{array}$ & $\begin{array}{r}\text { Polyethylene-co-vinyl acetate (PEVA) } \\
\text { Poly } n \text {-butyl methacrylate (PBMA) }\end{array}$ \\
\hline Espessura da cobertura & $0,6 \mu \mathrm{m}$ & $0,9 \mu \mathrm{m}$ \\
\hline $\begin{array}{l}\text { Espessura total das hastes } \\
\text { (haste cobertura/polímero droga) }\end{array}$ & $106 \mu \mathrm{m}^{*}$ & $154 \mu \mathrm{m}^{*}$ \\
\hline
\end{tabular}

A pré-dilatação da lesão-alvo era obrigatória e realizada com cateteres-balão com diâmetro pelo menos 0,5 mm menor que o diâmetro de referência do vaso e extensão menor que a do stent escolhido. O implante dos stents era realizado conforme as técnicas padrão vigentes, com ênfase para a cobertura total da lesão. Os stents disponíveis tinham diâmetro de $3,0 \mathrm{~mm}$ e 3,5 mm e extensão de 18 mm (Cypher ${ }^{\text {TM}}$ ) e 19 mm (VESTAsync ${ }^{\text {TM}}$ ). Era recomendado o uso de apenas um stent por lesão. Caso fosse necessário implante de novo stent, este deveria ser posicionado com pelo menos $2 \mathrm{~mm}$ de sobreposição de suas hastes ao primeiro stent. A pós-dilatação das endopróteses não era obrigatória, sendo recomendada nos casos com lesão residual maior que 20\% intrastent.

Seguimento invasivo, com angiografia e ultrassom intracoronariano, foi programado para todos os pacientes aos 4 e 12 meses após o procedimento.

\section{Angiografia coronariana quantitativa}

Angiografia do vaso-alvo foi realizada pré e pósprocedimento após administração de $100 \mu \mathrm{g}$ a $200 \mu \mathrm{g}$ de nitroglicerina intracoronariana, em duas projeções ortogonais. As mesmas angulações foram repetidas nos dois reestudos angiográficos. As imagens foram armazenadas em formato digital e analisadas utilizando-se sistema de detecção automática de bordas (QCA-CMS, Medis Medical Imaging System, Nuenem, Holanda).

Análise morfológica da lesão foi feita de acordo com os critérios padrão e a definição de sua complexidade, de acordo com o sistema de classificação modificado do American College of Cardiology/American Heart Association ${ }^{23}$.
A ponta do cateter-guia, preenchida por contraste, foi utilizada para calibração.

A análise quantitativa incluía mensuração dos seguintes parâmetros: 1) diâmetro de referência do vaso; 2) diâmetro luminal mínimo; 3) extensão da lesão; 4) porcentual do diâmetro de estenose (diâmetro de referência - diâmetro luminal mínimo/diâmetro de referência x 100); 5) ganho agudo (diâmetro luminal mínimo pós-procedimento - diâmetro luminal mínimo pré-procedimento); e 6) perda tardia (diâmetro luminal mínimo pós-procedimento - diâmetro luminal mínimo no seguimento). Reestenose binária foi definida como diâmetro de estenose $\geq 50 \%$.

\section{Análise do ultrassom intracoronariano}

As imagens do ultrassom intracoronariano pósprocedimento e nos períodos de seguimento foram adquiridas utilizando-se transdutor de elemento único rotacional, com frequência de $40 \mathrm{MHz}$, envolto em uma bainha com perfil 2,6 Fr, com recuo motorizado em sistema de tração automática à velocidade de $0,5 \mathrm{~mm} /$ segundo e conectado a um escâner comercial (CVIS, Galaxy $2^{\mathrm{TM}}$ ou iLab ${ }^{\mathrm{TM}}$, Boston Scientific Corp., Natick, Estados Unidos).

As imagens foram digitalizadas para análise quantitativa off-line de acordo com os critérios do American College of Cardiology's Clinical Expert Consensus Document on IVUS ${ }^{24}$.

Para realização da análise volumétrica, foi feita reconstrução tridimensional das imagens do ultrassom intracoronariano com a utilização de um programa de 
planimetria computadorizada comercialmente disponível (EchoPlaque 3.0, INDEC Systems Inc., Mountain View, Estados Unidos). As áreas do lúmen, do stent e do vaso (membrana elástica externa) foram determinadas a cada milímetro por planimetria computadorizada. A área de hiperplasia neointimal era calculada como a área do stent menos a área do lúmen. A partir de então, os volumes (do lúmen, do stent e do vaso) foram calculados utilizando-se os princípios da regra de Simpson ${ }^{24}$. O porcentual de obstrução volumétrica intrastent foi calculado como a razão entre o volume de hiperplasia neointimal e o volume do stent x 100 . Por fim, avaliamos também a aposição das hastes do stent à parede do vaso. Aposição incompleta foi definida como a separação de pelo menos uma haste da parede vascular, com presença de fluxo de sangue por trás das mesmas, sem envolver uma área de bifurcação com outro ramo ${ }^{25}$. A aposição incompleta foi ainda classificada em: 1) persistente (presente após o procedimento e no seguimento); 2) resolvida (presente após o procedimento e não mais identificada no seguimento) e 3) adquirida (ausente após o procedimento e presente no seguimento) ${ }^{26}$.

\section{Objetivos e desfechos do estudo}

Este estudo visa a avaliar se a ausência do polímero no stent VESTAsync ${ }^{\mathrm{TM}}$, liberador de sirolimus, compromete sua efetividade, comparando seus resultados com os do stent Cypher ${ }^{\mathrm{TM}}$, utilizado em uma coorte histórica de pacientes pareados para as mesmas características clínicas e angiográficas.

Nesse sentido, utilizamos dois parâmetros como desfechos primários de efetividade nesse estudo: a perda tardia pela angiografia e o porcentual de obstrução volumétrica intrastent gerado pela hiperplasia neointimal, avaliado pelo ultrassom intracoronariano.

Embora este estudo não tenha poder para avaliar a ocorrência de eventos clínicos, apresentamos também as taxas de ocorrência de eventos cardíacos adversos maiores: morte, infarto do miocárdio e necessidade de nova revascularização da lesão-alvo.

Todos os óbitos foram considerados de origem cardíaca, a menos que outra causa fosse identificada.

Infarto do miocárdio foi definido como elevação maior que duas vezes dos níveis de CK, com níveis da isoforma MB acima do valor de referência do laboratório. A associação com o aparecimento de ondas Q patológicas (mais de 0,04 segundo em duas ou mais derivações contíguas) no eletrocardiograma caracterizou infarto com onda Q.

Revascularização da lesão-alvo foi definida como nova revascularização (por intervenção percutânea ou cirurgia) do segmento previamente tratado, motivada por ocorrência de reestenose. Esse procedimento só era recomendado em caso de evidência de isquemia (piora ou novo aparecimento de angina ou isquemia detectada em provas funcionais), com novo estudo angiográfico demonstrando diâmetro de estenose intrastent $\geq 50 \%$.

\section{Análise estatística}

As análises foram realizadas com o programa SPSS 13.0 para Windows. Na análise descritiva, as variáveis categóricas foram expressas como frequências absolutas e porcentuais. As variáveis contínuas foram expressas como média \pm desvio padrão. O teste de quiquadrado e o teste exato de Fisher foram usados para comparação das variáveis categóricas. O teste $t$ de Student bicaudal foi usado para comparação das variáveis contínuas. Valores de $\mathrm{P}<0,05$ foram considerados estatisticamente significantes.

\section{RESULTADOS}

A população total estudada consistiu de 30 pacientes. A média das idades foi de 60,8 anos, $60 \%$ dos pacientes eram do sexo masculino, 30\% eram diabéticos e $43 \%$ apresentavam história prévia de infarto do miocárdio. Todos os pacientes eram portadores de síndromes coronarianas estáveis. A artéria descendente anterior foi o vaso mais frequentemente tratado nos dois grupos e a maioria das lesões foi considerada complexa pela classificação do American College of Cardiology/American Heart Association.

Os pacientes alocados no grupo VESTAsync ${ }^{\mathrm{TM}}$ eram portadores de vasos com diâmetro de referência significativamente menor $(2,67 \pm 0,32 \mathrm{~mm}$ vs. $2,98 \pm 0,4 \mathrm{~mm}$; $\mathrm{P}=0,02)$. Os pacientes do grupo Cypher ${ }^{\mathrm{TM}}$ possuíam lesões mais extensas $(12,9 \pm 1,9 \mathrm{~mm}$ vs. 9,98 \pm $1,98 \mathrm{~mm} ; \mathrm{P}=0,0003)$. As características clínicas e angiográficas de base são apresentadas na Tabela 2.

Conforme determinado pelo protocolo do estudo, todos os pacientes foram submetidos a pré-dilatação das lesões-alvo. A pós-dilatação foi realizada em 46,6\% dos pacientes do grupo VESTAsync ${ }^{\mathrm{TM}}$ e em $73 \%$ dos pacientes do grupo Cypher ${ }^{\mathrm{TM}}$. Nenhum paciente necessitou mais de um stent por lesão. A pressão de insuflação máxima foi significativamente maior nos pacientes do grupo Cypher $^{\mathrm{TM}}(14,8 \pm 2,3$ atm vs. 12,4 \pm 2,3 atm; $\mathrm{P}=0,008)$. Sucesso tanto angiográfico como do procedimento foi obtido em todos os pacientes.

No seguimento clínico de 12 meses, não houve ocorrência de nenhum caso de óbito, infarto do miocárdio ou necessidade de nova revascularização em qualquer dos pacientes, nos dois grupos. Trombose das endopróteses não foi identificada em nenhum paciente, nos dois grupos, até o seguimento de 12 meses.

\section{Angiografia coronariana quantitativa}

Análise angiográfica quantitativa foi realizada em todos os pacientes e é apresentada na Tabela 3.

Após o procedimento, obteve-se porcentual de estenose residual bem aceitável nos dois grupos (abai- 
TABELA 2

Características clínicas e angiográficas de base

\begin{tabular}{|c|c|c|c|}
\hline Característica & $\begin{array}{l}\text { VESTAsync }^{\mathrm{TM}} \\
\quad(\mathbf{n}=15)\end{array}$ & $\begin{array}{l}\text { Cypher TM } \\
(\mathbf{n}=15)\end{array}$ & Valor de $\mathbf{P}$ \\
\hline Idade, anos (média \pm DP) & $63,8 \pm 11,4$ & $57,9 \pm 10$ & 0,14 \\
\hline Sexo masculino, n (\%) & $9(60)$ & $9(60)$ & $>0,99$ \\
\hline Hipertensão arterial, n (\%) & $9(60)$ & $11(73,3)$ & 0,69 \\
\hline Dislipidemia, n (\%) & $7(46,6)$ & $8(53,3)$ & $>0,99$ \\
\hline Diabetes melito, n (\%) & $5(33,3)$ & $4(26,6)$ & $>0,99$ \\
\hline Tabagismo atual, n (\%) & $7(46,6)$ & $7(46,6)$ & $>0,99$ \\
\hline Infarto prévio, n (\%) & $7(46,6)$ & $6(40)$ & $>0,99$ \\
\hline Angina estável, n (\%) & $15(100)$ & $15(100)$ & $>0,99$ \\
\hline CRVM prévia, n (\%) & $2(13,3)$ & 0 & 0,48 \\
\hline \multicolumn{4}{|l|}{ Vaso-alvo, n (\%) } \\
\hline Artéria descendente anterior & $7(46,6)$ & $10(66,6)$ & 0,46 \\
\hline Artéria circunflexa & $4(26,6)$ & $2(13,3)$ & 0,65 \\
\hline Artéria coronária direita & $4(26,6)$ & $3(20)$ & $>0,99$ \\
\hline Lesões tipo B2/C*, n (\%) & $11(73,3)$ & $11(73,3)$ & $>0,99$ \\
\hline Diâmetro de referência do vaso, mm (média \pm DP) & $2,67 \pm 0,32$ & $2,98 \pm 0,4$ & 0,02 \\
\hline DLM, mm (média \pm DP) & $0,98 \pm 0,29$ & $1,16 \pm 0,3$ & 0,10 \\
\hline Extensão da lesão, mm (média \pm DP) & $9,98 \pm 1,98$ & $12,9 \pm 1,9$ & 0,0003 \\
\hline Diâmetro da estenose, \% (média \pm DP) & $63,5 \pm 9,90$ & $62 \pm 7$ & 0,63 \\
\hline
\end{tabular}

* Segundo a classificação do American College of Cardiology/American Heart Association.

CRVM = cirurgia de revascularização do miocárdio; $\mathrm{DLM}=$ diâmetro luminal mínimo; $\mathrm{DP}=$ desvio padrão; $\mathrm{n}=$ número de pacientes.

TABELA 3

Angiografia coronariana quantitativa

\begin{tabular}{|c|c|c|c|}
\hline Variáveis & $\begin{array}{l}\text { VESTAsync }^{\mathrm{TM}} \\
\quad(\mathbf{n}=15)\end{array}$ & $\begin{array}{l}\text { Cypher }^{\mathrm{TM}} \\
(\mathbf{n}=15)\end{array}$ & Valor de $\mathrm{P}$ \\
\hline \multicolumn{4}{|l|}{ Pós-procedimento } \\
\hline DLM intrastent, mm (média \pm DP) & $2,64 \pm 0,31$ & $2,94 \pm 0,44$ & 0,03 \\
\hline Estenose intrastent, $\%$ (média \pm DP) & $8,4 \pm 4,3$ & $5,09 \pm 6,72$ & 0,11 \\
\hline Ganho agudo, mm (média \pm DP) & $1,66 \pm 0,34$ & $1,74 \pm 0,38$ & 0,54 \\
\hline \multicolumn{4}{|l|}{ Seguimento 4 meses } \\
\hline DLM intrastent, mm (média \pm DP) & $2,34 \pm 0,33$ & $2,90 \pm 0,50$ & 0,0012 \\
\hline Estenose intrastent, \% (média \pm DP) & $13,8 \pm 7,0$ & $5,04 \pm 6,70$ & 0,0016 \\
\hline Perda tardia intrastent, mm (média \pm DP) & $0,30 \pm 0,25$ & $0,10 \pm 0,30$ & 0,06 \\
\hline \multicolumn{4}{|l|}{ Seguimento 12 meses } \\
\hline DLM intrastent, mm (média \pm DP) & $2,27 \pm 0,32$ & $2,63 \pm 0,46$ & 0,01 \\
\hline Estenose intrastent, \% (média \pm DP) & $12,9 \pm 8,9$ & $8,13 \pm 3,35$ & 0,06 \\
\hline Perda tardia intrastent, mm (média \pm DP) & $0,37 \pm 0,24$ & $0,16 \pm 0,36$ & 0,074 \\
\hline
\end{tabular}

xo de $10 \%$ ). O ganho agudo também foi semelhante nos dois grupos $\left(1,66 \pm 0,34 \mathrm{~mm}\right.$ para o VESTAsync ${ }^{\mathrm{TM}}$ vs. 1,74 $\pm 0,38 \mathrm{~mm}$ para o Cypher $\left.^{\mathrm{TM}} ; \mathrm{P}=0,54\right)$.

No seguimento de 4 meses, houve tendência a menor perda tardia intrastent no grupo Cypher ${ }^{\text {TM }}(0,10$ $\pm 0,30 \mathrm{~mm}$ vs. $0,30 \pm 0,25 \mathrm{~mm} ; \mathrm{P}=0,06)$, que se manteve no seguimento mais tardio $(0,16 \pm 0,36 \mathrm{~mm}$ vs. $0,37 \pm 0,24 \mathrm{~mm} ; P=0,074)$. A Figura 3 apresenta as curvas de distribuição da perda tardia, nos dois períodos de seguimento, em todos os pacientes dos dois grupos. 


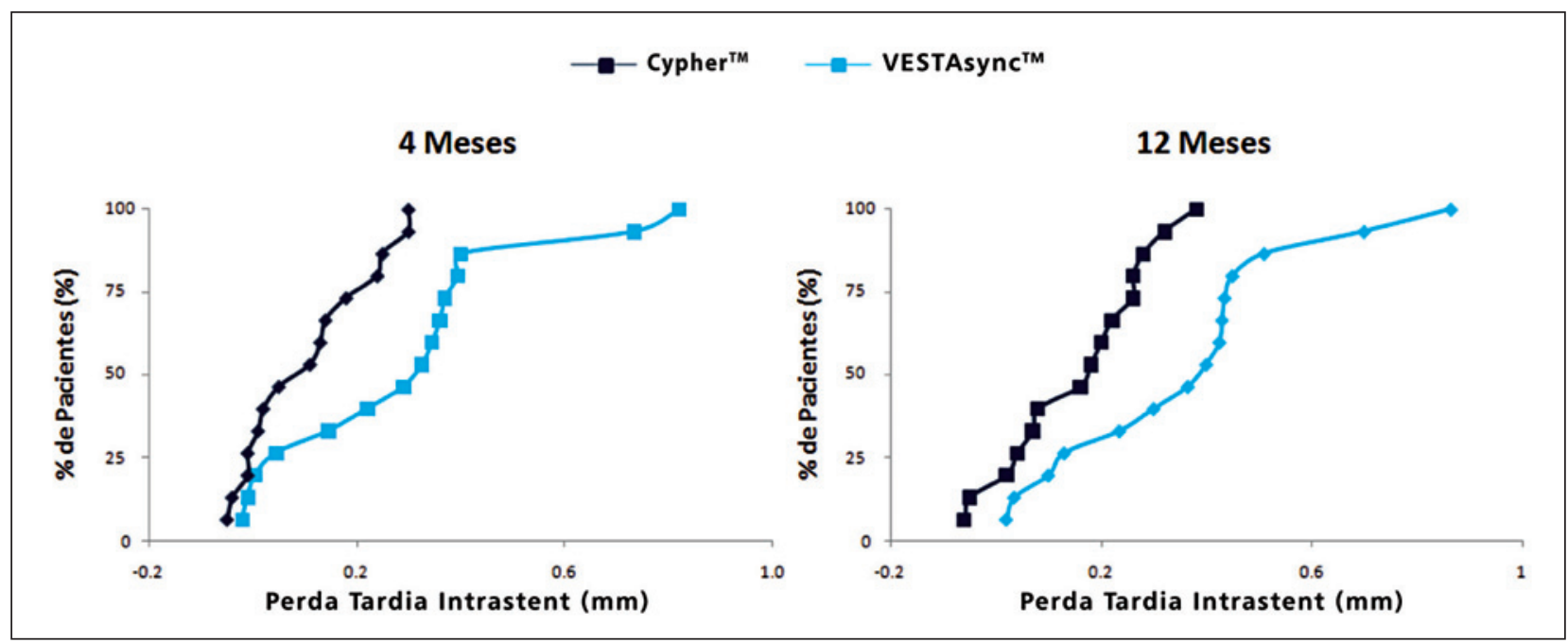

Figura 3 - Curvas de distribuição da perda tardia para os stents Cypher $^{\mathrm{TM}}$ e VESTAsync ${ }^{\mathrm{TM}}$. Observa-se, no grupo Cypher ${ }^{\mathrm{TM}}$, que a maioria dos pacientes apresentou perda tardia abaixo de $0,2 \mathrm{~mm}$ aos 4 meses, com pequeno desvio para a direita na avaliação de 12 meses. No grupo VESTAsync ${ }^{T M}$, a curva é mais abrangente, identificando-se dois pacientes fora do padrão (outliers) desde os 4 meses, com perda tardia, respectivamente, de 0,73 mm e 0,82 mm. Aos 12 meses, percebe-se pequeno desvio da curva para a direita, sem adição de caso adicional com perda tardia elevada.

Reestenose binária não foi identificada em qualquer dos grupos até o seguimento avaliado.

\section{Ultrassom intracoronariano}

Análise volumétrica com ultrassom intracoronariano foi realizada em todos os pacientes dos dois grupos e é apresentada na Tabela 4.

Nos dois períodos de seguimento, não houve diferença significativa quanto a volume do vaso, stent ou lúmen entre os dois grupos.

Aos 4 meses, o volume de hiperplasia neointimal produzido foi semelhante nos dois grupos $(4,3 \pm$ 3,5 $\mathrm{mm}^{3}$ para o VESTAsync ${ }^{\mathrm{TM}}$ vs. 3,0 $\pm 4,8 \mathrm{~mm}^{3}$ para o Cypher $\left.{ }^{\text {TM }} ; P=0,40\right)$. O porcentual de obstrução volumétrica do stent por esse volume de hiperplasia também foi similar $\left(2,8 \pm 2,2 \%\right.$ para o VESTAsync ${ }^{\mathrm{TM}} \mathrm{vs}$. $2,2 \pm 3,8 \%$ para o Cypher $\left.{ }^{\mathrm{TM}} ; \mathrm{P}=0,60\right)$.

Entretanto, no segundo reestudo invasivo, notouse acúmulo maior de hiperplasia no grupo VESTAsync ${ }^{\mathrm{TM}}$, ao passo que houve um "congelamento" do resultado no grupo Cypher" (volume de hiperplasia: 6,1 $\pm 4,2 \mathrm{~mm}^{3}$ vs. $\left.3,0 \pm 4,5 \mathrm{~mm}^{3} ; \mathrm{P}=0,07\right)$, gerando porcentual de obstrução volumétrica do stent de 4,0 $\pm 2,2 \%$ para o grupo VESTAsync ${ }^{\mathrm{TM}}$ e de $2,4 \pm 3,9 \%$ para o grupo Cypher ${ }^{\mathrm{TM}}(\mathrm{P}=0,17)$ (Figura 4).

Ademais, observou-se no grupo VESTAsync ${ }^{\mathrm{TM}}$ dois casos de má aposição das hastes do stent imediatamente após o procedimento, que se resolveram no seguimento de 4 meses. Não houve surgimento de nenhum caso de má aposição de hastes adquirida nesse grupo. Nos pacientes tratados com o stent Cypher ${ }^{\mathrm{TM}}$, observou-se um caso de má aposição após o procedimen- to, resolvida no seguimento de 4 meses, e o surgimento de um caso adicional de má aposição adquirida aos 4 meses, que não progrediu até a última avaliação ultrassonográfica.

\section{DISCUSSÃo}

O principal achado deste estudo é que a ausência do polímero no stent VESTAsync ${ }^{\mathrm{TM}}$, liberador de sirolimus, não influenciou sua eficácia. Além disso, não foram observados sinais de toxicidade importante à parede do vaso, com ausência de remodelamento vascular positivo e má aposição adquirida de hastes do stent.

A eficiência na redução da hiperplasia neointimal pelos stents farmacológicos de primeira geração, notadamente Cypher ${ }^{\mathrm{TM}}$ e Taxus ${ }^{\mathrm{TM}}$ (Boston Scientific Corp., Natick, Estados Unidos), é inequívoca ${ }^{1-4}$. No entanto, relatos de trombose tardia desses dispositivos têm trazido questionamentos recentes a sua segurança ${ }^{6-8}$.

A presença de um polímero sintético, componente essencial nesses dispositivos de primeira geração e responsável pelo carreamento e pela liberação das drogas antiproliferativas, tem sido relacionada à produção de intensa resposta inflamatória ${ }^{10-12,27}$. De fato, estudos em animais têm demonstrado remodelamento vascular positivo exacerbado, possivelmente como consequência de reação inflamatória local, em resposta à presença do polímero em contato com a parede vascular $^{28}$. Mais recentemente, foi demonstrada associação entre remodelamento vascular positivo e ocorrência de trombose tardia de stents farmacológicos ${ }^{25,26}$.

A ocorrência desses fatos tem motivado o desenvolvimento de novas tecnologias, com polímeros mais 
TABELA 4

Análise volumétrica pelo ultrassom intracoronariano

\begin{tabular}{|c|c|c|c|}
\hline Variáveis & $\begin{array}{l}\text { VESTAsync }^{\mathrm{TM}} \\
(\mathbf{n}=\mathbf{1 5})\end{array}$ & $\begin{array}{l}\text { Cypher }^{\mathrm{TM}} \\
(\mathrm{n}=15)\end{array}$ & Valor de $P$ \\
\hline \multicolumn{4}{|l|}{ Pós-procedimento } \\
\hline Volume do vaso, $\mathrm{mm}^{3}$ (média $\left.\pm \mathrm{DP}\right)$ & $294,2 \pm 117,1$ & $302,4 \pm 118$ & 0,84 \\
\hline Volume do stent, $\mathrm{mm}^{3}$ (média $\pm \mathrm{DP}$ ) & $144,5 \pm 48,2$ & $152,2 \pm 40$ & 0,63 \\
\hline 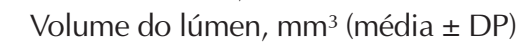 & $144,7 \pm 48,4$ & $152,2 \pm 40$ & 0,64 \\
\hline \multicolumn{4}{|l|}{ Seguimento 4 meses } \\
\hline Volume do vaso, $\mathrm{mm}^{3}$ (média $\pm \mathrm{DP}$ ) & $286,9 \pm 87,4$ & $305,6 \pm 121$ & 0,63 \\
\hline Volume do stent, $\mathrm{mm}^{3}$ (média $\pm \mathrm{DP}$ ) & $140,5 \pm 36,7$ & $154,4 \pm 44$ & 0,35 \\
\hline 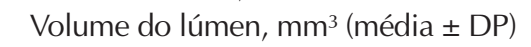 & $136,3 \pm 34,2$ & $137,6 \pm 40$ & 0,92 \\
\hline Volume da HNI, mm³ (média \pm DP) & $4,3 \pm 3,5$ & $3,0 \pm 4,8$ & 0,40 \\
\hline $\begin{array}{l}\text { Obstrução volumétrica pela HNI, \% } \\
\text { (média } \pm \text { DP) }\end{array}$ & $2,8 \pm 2,2$ & $2,2 \pm 3,8$ & 0,60 \\
\hline \multicolumn{4}{|l|}{ Seguimento 12 meses } \\
\hline Volume do vaso, $\mathrm{mm}^{3}$ (média $\left.\pm \mathrm{DP}\right)$ & $288,4 \pm 81,3$ & $304,3 \pm 89,6$ & 0,61 \\
\hline Volume do stent, $\mathrm{mm}^{3}$ (média $\pm \mathrm{DP}$ ) & $140,7 \pm 38,9$ & $150,6 \pm 59,4$ & 0,59 \\
\hline Volume do lúmen, $\mathrm{mm}^{3}$ (média $\pm \mathrm{DP}$ ) & $134,8 \pm 35,1$ & $147,7 \pm 60,7$ & 0,48 \\
\hline Volume da HNI, mm³ (média \pm DP) & $6,1 \pm 4,2$ & $3,0 \pm 4,5$ & 0,07 \\
\hline $\begin{array}{l}\text { Obstrução volumétrica pela HNI, \% } \\
\text { (média } \pm \text { DP) }\end{array}$ & $4,0 \pm 2,2$ & $2,4 \pm 3,9$ & 0,17 \\
\hline
\end{tabular}

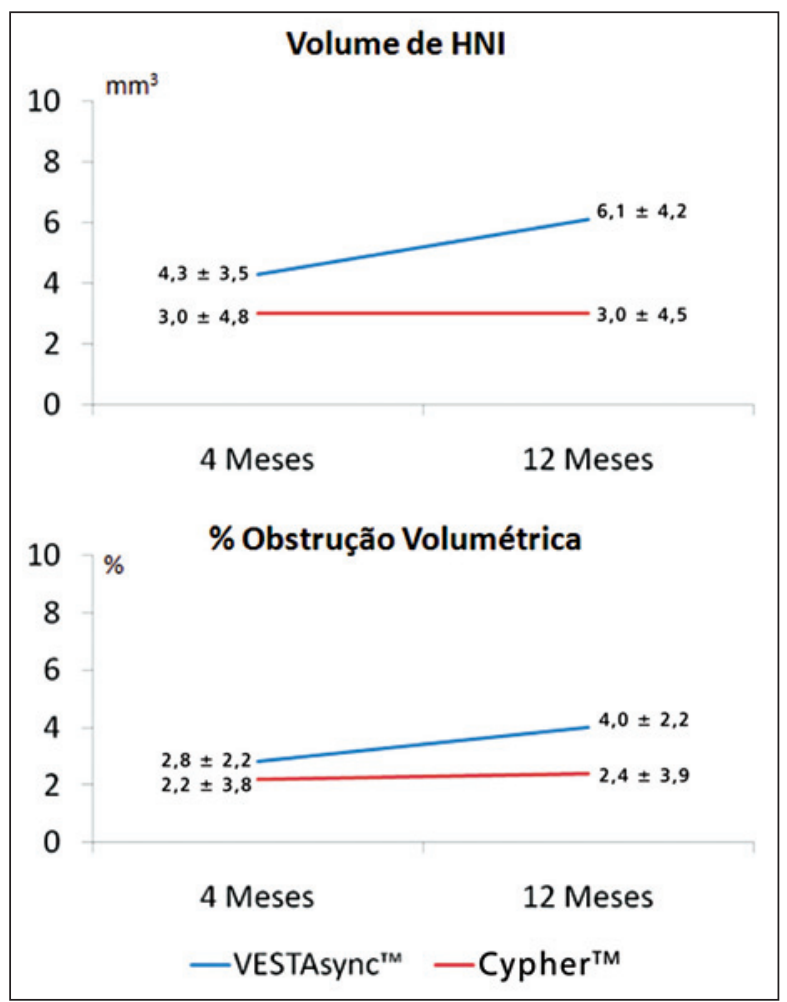

Figura 4 - Análise volumétrica da hiperplasia neointimal (HNI) e porcentual de obstrução pelo ultrassom intracoronariano. Observase discreto aumento dos volumes de hiperplasia e porcentual de obstrução dos 4 para 12 meses no grupo VESTAsync ${ }^{\top M}$, ao passo que há um "congelamento" do resultado no grupo Cypher ${ }^{\mathrm{TM}}$. biocompatíveis e menos tóxicos à parede vascular, e até mesmo tecnologias de liberação dos fármacos sem a necessidade da cobertura polimérica.

O grande desafio tem sido aumentar a segurança dessas endopróteses sem comprometer sua eficácia antiproliferativa. No entanto, a retirada do polímero da composição dos stents farmacológicos pode comprometer sobremaneira sua eficácia, uma vez que é o componente responsável não só pelo carreamento da droga, mas também por controlar sua cinética de liberação.

Avaliação com ultrassom intracoronariano do estudo ASian Paclitaxel-Eluting Stent Clinical Trial (ASPECT) ${ }^{13}$ demonstrou que stents recobertos com formulação não-polimérica de paclitaxel foram eficazes em reduzir a hiperplasia neointimal, após 6 meses do implante, quando comparados ao grupo controle com stents não-farmacológicos. Os autores demonstraram ainda que os efeitos foram dose-dependente, com aumento da supressão intimal com o incremento da dose do paclitaxel [volume de hiperplasia neointimal: $31 \pm 22 \mathrm{~mm}^{3}$ no controle; $18 \pm 15 \mathrm{~mm}^{3}$ no paclitaxel baixa dose $\left(1,28 \mu \mathrm{g} / \mathrm{mm}^{2}\right) ; 13 \pm 14 \mathrm{~mm}^{3}$ no paclitaxel alta dose $\left.\left(3,10 \mu \mathrm{g} / \mathrm{mm}^{2}\right) ; \mathrm{P}<0,001\right]$. No entanto, aos dois anos, esses benefícios foram anulados, com aumento do acúmulo da hiperplasia neointimal dos 6 meses para dois anos de $13 \pm 14 \mathrm{~mm}^{3}$ no grupo de alta dose, comparativamente a $4 \pm 7 \mathrm{~mm}^{3}$ no grupo de baixa dose $(P=0,074)$ e a $1 \pm 13 \mathrm{~mm}^{3}$ no grupo controle $(P=0,019)$, configurando o fenômeno de late catch-up. 
Da mesma forma, no estudo DELIVER ${ }^{14}$, o stent ACHIEVE'M com liberação não-polimérica de paclitaxel foi comparado ao stent Multi-Link Penta ${ }^{\text {TM, }}$, apresentando menor formação neointimal (perda tardia de $0,81 \mathrm{~mm}$ vs. $0,98 \mathrm{~mm} ; \mathrm{P}=0,003)$, mas sem alcançar os desfechos primário de falência do vaso-alvo $(11,9 \%$ vs. $14,5 \% ; P=0,12)$ e secundário de reestenose binária $(14,9 \%$ vs. $20,6 \% ; P=0,076)$.

Em estudo clínico que avaliou diversas doses da rapamicina, Hausleiter et al. ${ }^{15}$ demonstraram que a cobertura não-polimérica de stent coronariano com rapamicina é segura e leva à redução dose-dependente das taxas de reestenose.

No estudo ISAR-TEST ${ }^{16}$, do mesmo grupo, o stent Yukon $^{\mathrm{TM}}$ recoberto com solução de rapamicina a $2 \%$ não-polimérica foi comparado ao stent Taxus ${ }^{\top M}$, liberador de paclitaxel polimérico. O stent Yukon ${ }^{\mathrm{TM}}$ se mostrou não inferior, com perda tardia intrastent de 0,48 \pm $0,61 \mathrm{~mm}$ contra $0,48 \pm 0,58 \mathrm{~mm}$ para o stent Taxus $^{\mathrm{TM}}$ $(\mathrm{P}=0,98)$.

Entretanto, no estudo ISAR-TEST-3 ${ }^{17}, 605$ pacientes foram randomizados para tratamento com stents com eluição de sirolimus com polímero biodegradável ( $n=202)$, sem polímero $(n=201)$ e com polímero durável $\left(\right.$ Cypher $\left.^{\mathrm{TM}}, \mathrm{n}=202\right)$. No seguimento angiográfico de 6 a 8 meses, os stents com polímero biodegradável tiveram eficácia semelhante à do Cypher ${ }^{\mathrm{TM}}$ (perda tardia de 0,17 $\pm 0,45 \mathrm{~mm}$ vs. 0,23 $\pm 0,46 \mathrm{~mm}$ ), atingindo o critério de não-inferioridade $(\mathrm{P}<0,001)$. No entanto, o stent não-polimérico apresentou perda tardia superior $(0,47 \pm 0,56 \mathrm{~mm})$, não atingindo a margem de não-inferioridade em comparação ao Cy$\operatorname{pher}^{\text {TM }}(P=0,94)$.

O stent VESTAsync ${ }^{\top M}$, avaliado neste trabalho, agrega algumas características dignas de nota.

Primeiramente, dispensa a necessidade de um polímero sintético, apresentando modificação da superfície metálica das hastes do stent, recobertas com hidroxiapatita, substância que constitui cerca de $70 \%$ da composição natural dos ossos humanos e que assume o papel de carreadora e controladora da liberação da droga. Não promove reação inflamatória ou resposta de corpo estranho, contribuindo para sua excelente biocompatibilidade ${ }^{19,20}$, já confirmada em estudos clínicos ${ }^{21}$.

Outro ponto que merece consideração é a dose do fármaco empregada no stent VESTAsync ${ }^{T M}$. Tem sido sugerido que o tipo e a dose do fármaco antiproliferativo sejam outros fatores implicados na toxicidade dos stents farmacológicos. Estudo de Nakamura et al. ${ }^{29}$ demonstrou ser possível manter a mesma efetividade do stent Cypher ${ }^{\mathrm{TM}}$ utilizando-se $70 \%$ ou mesmo $45 \%$ da dose original do sirolimus. No stent VESTAsync ${ }^{T M}$, o sirolimus é utilizado em uma formulação lipofílica, que impregna as microporosidades da hidroxiapatita, não aumentando a espessura da cobertura. A dose utilizada é de $55 \mu \mathrm{g}$ no stent de $19 \mathrm{~mm}$ de extensão, $65 \%$ menor que a utilizada no stent Cypher ${ }^{\mathrm{TM}}(140 \mu \mathrm{g}$ no stent de $18 \mathrm{~mm}$ ). Além disso, sua cinética de liberação é mais lenta, aproximando-se àquela do Cypher ${ }^{\mathrm{TM}}$ na primeira hora, caindo para a metade a partir de então, com cerca de $80 \%$ da droga liberada ao final de 60 dias (Figura 2; Tabela 1).

O presente estudo visa à apresentação dos resultados angiográficos e ultrassonográficos desse stent farmacológico de nova geração, colocados em perspectiva contra os do stent Cypher ${ }^{\mathrm{TM}}$, considerado o stent farmacológico padrão quando se pensa em eficácia antiproliferativa.

Observamos que, já desde os 4 meses, na análise angiográfica, o stent VESTAsync ${ }^{\top M}$ apresenta perda luminal tardia três vezes maior que a do Cypher ${ }^{\mathrm{TM}}$ $(0,30 \pm 0,25 \mathrm{~mm}$ vs. $0,10 \pm 0,30 \mathrm{~mm} ; \mathrm{P}=0,06)$. Isso se explica pela maior perda tardia observada em dois casos $(0,73 \mathrm{~mm}$ e $0,82 \mathrm{~mm})$, que numa amostra de apenas 15 pacientes eleva a média da população e contribui para essa diferença. Esse fenômeno está bem demonstrado na Figura 3. Esses achados estão em discordância com o porcentual de obstrução volumétrica mensurado pelo ultrassom intracoronariano, que foi bastante similar entre os dois grupos. Uma possível explicação para essa diferença poderia ser a falência focal na inibição da hiperplasia neointimal.

No seguimento mais tardio, o stent Cypher ${ }^{\mathrm{TM}}$ manteve seu resultado praticamente "congelado", enquanto se observou, no stent VESTAsync ${ }^{\top M}$, um acúmulo de quantidade ligeiramente maior de hiperplasia (Figura 4). É possível que outros fatores, além do tempo de eluição do sirolimus, possam ter influenciado esse perfil de inibição da hiperplasia neointimal.

Mauri et al. ${ }^{30}$ propuseram um modelo matemático, que demonstrou aumento das taxas de reestenose binária com o aumento da perda luminal tardia. De acordo com esse modelo, o aumento da perda tardia de $0,2 \mathrm{~mm}$ para $0,4 \mathrm{~mm}$ resultaria em aumento das taxas de reestenose da ordem de 3,1\%. No entanto, caso a perda tardia aumente a partir de $0,4 \mathrm{~mm}$ para $0,6 \mathrm{~mm}$, há incremento duas vezes maior da reestenose binária, da ordem de 6,4\%.

A perda tardia apresentada pelo stent VESTAsync ${ }^{\text {TM }}$ até o seguimento de 12 meses, abaixo de $0,4 \mathrm{~mm}$, posiciona esse stent de nova geração, não polimérico, dentre os stents farmacológicos de maior efetividade, sem que isso tenha se traduzido em aumento das taxas de reestenose e/ou necessidade de nova revascularização.

Seguimento mais tardio, com maior número de pacientes e casos de maior complexidade, é necessário para avaliar o impacto que essa nova tecnologia possa causar no quesito segurança. 


\section{Limitações}

A natureza não-randomizada desta análise poderia contribuir para a introdução de erros de seleção. Tentamos minimizar essa possibilidade selecionando uma população controle com as mesmas características clínicas e que tivessem sido tratadas sob os mesmos critérios de inclusão e exclusão.

O pequeno número da amostra impede avaliação definitiva de desfechos clínicos. No entanto, no que se refere à análise de eficácia, o uso da angiografia coronariana quantitativa e do ultrassom intracoronariano, de forma seriada, fornece informações suficientes para análise desse desfecho, bem como para entendimento dos mecanismos de ação dos tipos de dispositivo estudados.

O tempo relativamente curto de acompanhamento, associado ao pequeno número da amostra e à inclusão de casos de moderada a baixa complexidades, impedem conclusões mais definitivas com respeito à segurança desse novo dispositivo.

\section{CONCLUSÕES}

A ausência de polímero e a menor dose do sirolimus na composição do novo stent $V_{E S T A s y n c}{ }^{T M}$ não comprometeram significativamente sua eficácia antiproliferativa até o seguimento de um ano, situando esse novo stent de terceira geração dentre os que têm maior potência na inibição da formação da hiperplasia neointimal.

Tempo mais longo de acompanhamento e inclusão de casos de maior complexidade são necessários para se avaliar o impacto dessas inovações em seu perfil de segurança.

\section{CONFLITO DE INTERESSES}

Os autores declararam inexistência de conflito de interesses.

\section{REFERÊNCIAS BIBLIOGRÁFICAS}

1. Sousa JE, Costa MA, Abizaid AC, Rensing BJ, Abizaid AS, Tanajura LF, et al. Sustained suppression of neointimal proliferation by sirolimus-eluting stents: one-year angiographic and intravascular ultrasound follow-up. Circulation. 2001; 104(17):2007-11.

2. Morice MC, Serruys PW, Sousa JE, Fajadet J, Ban Hayashi E, Perin $M$, et al. Randomized study with the sirolimus-coated bx velocity balloon-expandable stent in the treatment of patients with de novo native coronary artery lesions. A randomized comparison of a sirolimus-eluting stent with a standard stent for coronary revascularization. RAVEL Study Group. N Engl J Med. 2002;346(23):1773-80.

3. Moses JW, Leon MB, Popma JJ, Fitzgerald PJ, Holmes DR, $\mathrm{O}^{\prime}$ Shaughnessy $\mathrm{C}$, et al. Sirolimus-eluting stents versus standard stents in patients with stenosis in a native coronary artery. SIRIUS Investigators. N EngI J Med. 2003;349(14): 1315-23.

4. Stone GW, Ellis SG, Cox DA, Hermiller J, O'Shaughnessy C, Mann JT, et al. A polymer-based, paclitaxel-eluting stent in patients with coronary artery disease. TAXUS-IV Investigators. N Engl J Med. 2004;350(3):221-31.

5. Kereiakes DJ, Kuntz RE, Mauri L, Krucoff MW. Surrogates, substudies, and real clinical end points in trials of drugeluting stents. J Am Coll Cardiol. 2005;45(8):1206-12.

6. Feres F, Costa JR Jr, Abizaid A. Very late thrombosis after drugeluting stents. Catheter Cardiovasc Interv. 2006;68(1):83-8.

7. McFadden EP, Stabile E, Regar E, Cheneau E, Ong AT, Kinnaird $\mathrm{T}$, et al. Late thrombosis in drug-eluting stents after discontinuation of antiplatelet therapy. Lancet. 2004;364(9444): 1519-21.

8. Win HK, Caldera AE, Maresh K, Lopez J, Rihal CS, Parikh $M A$, et al. Clinical outcomes and stent thrombosis following off-label use of drug-eluting stents. EVENT Registry Investigators. JAMA. 2007;297(18):2001-9.

9. Wessely R, Kastrati A, Schömig A. Late restenosis in patients receiving a polymer-coated sirolimus-eluting stent. Ann Intern Med. 2005;143(5):392-4.

10. Virmani R, Liistro F, Stankovic G, Di Mario C, Montorfano M, Farb A, et al. Mechanism of late in-stent restenosis after implantation of a paclitaxel derivate-eluting polymer stent system in humans. Circulation. 2002;106(21):2649-51.

11. Virmani R, Guagliumi G, Farb A, Musumeci G, Grieco N, Motta $\mathrm{T}$, et al. Localized hypersensitivity and late coronary thrombosis secondary to a sirolimus-eluting stent: should we be cautious? Circulation. 2004;109(6):701-5.

12. Guagliumi G, Farb A, Musumeci G, Valsecchi O, Tespili M, Motta T, et al. Images in cardiovascular medicine. Sirolimuseluting stent implanted in human coronary artery for 16 months: pathological findings. Circulation. 2003;107(9):1340-1.

13. Hong MK, Mintz GS, Lee CW, Song JM, Han KH, Kang DH, et al. ASian Paclitaxel-Eluting Stent Clinical Trial. Paclitaxel coating reduces in-stent intimal hyperplasia in human coronary arteries: a serial volumetric intravascular ultrasound analysis from the ASian Paclitaxel-Eluting Stent Clinical Trial (ASPECT). Circulation. 2003;107(4):517-20.

14. Lansky AJ, Costa RA, Mintz GS, Tsuchiya Y, Midei M, Cox DA, et al. Non-polymer-based paclitaxel-coated coronary stents for the treatment of patients with de novo coronary lesions: angiographic follow-up of the DELIVER clinical trial. DELIVER Clinical Trial Investigators. Circulation. 2004; 109(16):1948-54.

15. Hausleiter J, Kastrati A, Wessely R, Dibra A, Mehilli J, Schratzenstaller $\mathrm{T}$, et al. Prevention of restenosis by a novel drug-eluting stent system with a dose-adjustable, polymerfree, on-site stent coating. Eur Heart J. 2005;26(15):1475-81.

16. Mehilli J, Kastrati A, Wessely R, Dibra A, Hausleiter J, Jaschke B, et al. Randomized trial of a nonpolymer-based rapamycin-eluting stent versus a polymer-based paclitaxeleluting stent for the reduction of late lumen loss. Intracoronary Stenting and Angiographic Restenosis - Test Equivalence Between 2 Drug-Eluting Stents (ISAR-TEST) Trial Investigators. Circulation. 2006;113(2):273-9.

17. Mehilli J, Byrne RA, Wieczorek A, lijima R, Schulz S, Bruskina $\mathrm{O}$, et al. Randomized trial of three rapamycin-eluting stents with different coating strategies for the reduction of coronary restenosis. Intracoronary Stenting and Angiographic Restenosis Investigators - Test Efficacy of Rapamycin-eluting Stents with Different Polymer Coating Strategies (ISAR-TEST-3). Eur Heart J. 2008;29(16):1975-82.

18. Costa Jr JR, Abizaid A, Costa R, Feres F, Tanajura LF, Abizaid AS, et al. Preliminary results of the hidroxyapatite nonpolymerbased sirolimus-eluting stent for the treatment of single de novo coronary lesions. A first-in-human analysis of a thirdgeneration drug-eluting stent system. J Am Coll Cardiol Intv. 2008;1:545-51. 
19. Eppley BL, Pietrzak WS, Blanto MW. Allograft and alloplastic bone substitutes: a review of science and technology for the craniomaxillofacial surgeon. J Craniofac Surg. 2005; 16(6):981-9.

20. Jarcho M. Retrospective analysis of hydroxyapatite development for oral implant applications. Dent Clin North Am. 1992;36(1):19-26.

21. Pezzatini S, Solito R, Morbidelli L, Lamponi S, Boanini E, Bigi $A$, et al. The effect of hydroxyapatite nanocrystals on microvascular endothelial cell viability and functions. J Biomed Mater Res A. 2006;76(3):656-63.

22. Groth CG, Bäckman L, Morales JM, Calne R, Kreis H, Lang $P$, et al. Sirolimus (rapamycin)-based therapy in human renal transplantation: similar efficacy and different toxicity compared with cyclosporine. Sirolimus European Renal Transplant Study Group. Transplantation. 1999;67(7):1036-42.

23. Ellis SG, Vandormael MG, Cowley MJ, DiSciascio G, Deligonul U, Topol EJ, et al. Coronary morphologic and clinical determinants of procedural outcome with angioplasty for multivessel coronary disease. Implications for patient selection. Multivessel Angioplasty Prognosis Study Group. Circulation. 1990;82(4): 1193-202.

24. Mintz GS, Nissen SE, Anderson WD, Bailey SR, Erbel R, Fitzgerald PJ, et al. American College of Cardiology Clinical Expert Consensus Document on Standards for Acquisition, Measurement and Reporting of Intravascular Ultrasound Studies (IVUS). A report of the American College of Cardiology Task
Force on Clinical Expert Consensus Documents. J Am Coll Cardiol. 2001;37(5):1478-92.

25. Cook S, Wenaweser $P$, Togni $M$, Billinger $M$, Morger $C$, Seiler $C$, et al. Incomplete stent apposition and very late stent thrombosis after drug-eluting stent implantation. Circulation. 2007;115(18):2426-34.

26. Siqueira DA, Abizaid AA, Costa JR, Feres F, Mattos LA, Staico $R$, et al. Late incomplete apposition after drug-eluting stent implantation: incidence and potential for adverse clinical outcomes. Eur Heart J. 2007;28(11):1304-9.

27. Lüscher TF, Steffel J, Eberli FR, Joner M, Nakazawa G, Tanner FC, et al. Drug-eluting stent and coronary thrombosis: biological mechanisms and clinical implications. Circulation. 2007;115(8):1051-8.

28. Nebeker JR, Virmani R, Bennett CL, Hoffman JM, Samore $\mathrm{MH}$, Alvarez J, et al. Hypersensitivity cases associated with drug-eluting coronary stents: a review of available cases from the Research on Adverse Drug Events and Reports (RADAR) project. J Am Coll Cardiol. 2006;47(1):175-81.

29. Nakamura M, Abizaid A, Hirohata A, Honda Y, Sousa JE, Fitzgerald PJ. Efficacy of reduced-dose sirolimus-eluting stents in human coronary artery: serial IVUS analysis of neointimal hyperplasia and luminal dimension. Catheter Cardiovasc Interv. 2007;70(7):946-51.

30. Mauri L, Orav EJ, Kuntz RE. Late loss in lumen diameter and binary restenosis for drug-eluting stent comparison. Circulation. 2005;111(25):3435-42. 